\title{
Use of Anticholinergic Drugs According to Various Criteria and Their Association With Psychological Well-Being and Mortality in Long-Term Care Facilities
}

\author{
Aalto, Ulla L.
}

2019-09

Aalto , U L , Finne-Soveri , H , Kautiainen , H , Roitto , H-M , Öhman , H \& Pitkälä , K H 2019

, ' Use of Anticholinergic Drugs According to Various Criteria and Their Association With

Psychological Well-Being and Mortality in Long-Term Care Facilities ' , Journal of the

American Medical Directors Association , vol. 20 , no. 9 , pp. 1156-1162 . https://doi.org/10.1016/j.jamda.2019.02.00

http://hdl.handle.net/10138/318484

https://doi.org/10.1016/j.jamda.2019.02.007

cc_by_nc_nd

acceptedVersion

Downloaded from Helda, University of Helsinki institutional repository.

This is an electronic reprint of the original article.

This reprint may differ from the original in pagination and typographic detail.

Please cite the original version. 

psychological well-being and mortality in long-term care facilities

3 Ulla L. Aalto, MD a,b; Harriet Finne-Soveri, MD, PhD ${ }^{\text {b,c }}$; Hannu Kautiainen, $\mathrm{PhD}{ }^{\mathrm{d}}$; Hanna-Maria 4 Roitto, MD ${ }^{\text {a,b }}$; Hannareeta Öhman, MD, PhD ${ }^{\text {a,b }}$; Kaisu H Pitkälä, MD, PhD ${ }^{\text {a,d }}$ 5 Services and Health Care, Helsinki Hospital, Helsinki, Finland; ${ }^{\mathrm{c}}$ National Institute for Health and Welfare, Helsinki, Finland; ${ }^{d}$ Helsinki University Hospital, Unit of Primary Health Care, Helsinki, Finland 2 3 4 15 6 17 8 9 20 1 
Objectives 1) To compare three internationally established criteria for drugs with anticholinergic properties (DAPs) and their associated factors in long-term care facilities. 2) To investigate the association between use of DAPs and psychological well-being (PWB) or mortality.

Design Cross-sectional study and 1-year follow-up of all-cause mortality.

Setting and Participants Of all 4449 residents living in long-term care facilities in Helsinki in 2011,2432 ( $\geq 65$ years of age) participated after exclusion of residents with severe dementia.

Measurements Data on demographics, medication use, and active diagnoses were collected by trained staff using structured questionnaires. DAP use was defined by the following three international criteria: Chew's list, the Anticholinergic Risk Scale (ARS), and the Anticholinergic Drug Scale (ADS). The total number of DAPs was counted and referred to as anticholinergic burden. PWB was assessed by a questionnaire and yielded a score ranging from 0 to 1 . Mortality data was retrieved from central registers.

Results Of all participants, $85 \%$ were DAP users according to at least one of the three criteria used. Overlap between the three criteria was only moderate. DAP users were younger and a larger proportion of them had better cognition. However, they suffered more often from depression and other psychiatric diagnoses than nonusers. DAP users had lower PWB scores than those not using DAPs, and PWB decreased linearly in the overlapping groups from nonusers to those using DAPs according to all three criteria. The total number of DAPs used predicted mortality.

Conclusions and Implications DAP use and PWB appear to be negatively associated. When combining several criteria of DAPs, their burden predicted mortality. Clinicians should carefully consider the potential benefits and harms when prescribing DAPs to older persons. 


\section{Introduction}

Drugs with anticholinergic properties (DAPs) are a heterogenous group of medicines. They are considered as potentially harmful to older people because of their adverse effects peripherally and especially on the nervous system. ${ }^{1}$ Some important peripheral effects of DAPs include dryness of mouth, tachycardia, constipation and blurred vision. ${ }^{2,3}$ Undesired central side-effects of DAPs include increased risk for functional decline,${ }^{4,5}$ falls, ${ }^{6}$ cognitive decline,${ }^{7-10}$ and increased use of health services. ${ }^{11,12}$ Central adverse effects are known to be dose-dependent. Accordingly, a higher anticholinergic burden is associated with a higher risk of adverse outcomes. ${ }^{5}$ DAPs are still widely used among frail older people living in residential care. ${ }^{13-15}$ DAPs are frequently used to manage various symptoms such as urinary incontinence, anxiety, and pain. ${ }^{16}$ The effect of DAPs on mortality has also been investigated but most studies have not shown a clear relationship between DAP use and mortality. ${ }^{6,13,17-19}$ However, there are a few studies showing contradictory findings. ${ }^{8,20,21}$

Residents in long-term care generally suffer from multiple chronic diseases and have deficits in functioning and cognition. Management should thus aim towards symptom relief and palliative care, and quality of life (QOL) should be one of the most important goals of care. Psychological well-being (PWB) is a means to evaluate one dimension of QOL. To our knowledge, few studies have investigated the association between DAPs and QOL. There is some evidence that residents using DAPs living in long-term care facilities also have lower PWB than those not using DAPs. ${ }^{15,22}$

Several lists classifying anticholinergic drugs and their anticholinergic properties have been developed. ${ }^{4,5}$ We aimed to compare these DAP lists with each other. To explore outcomes of DAPs, we chose the three most highly cited lists used internationally according to Web of Science and Google Scholar. ${ }^{5,23-25}$ These three lists were created in various ways. While Chew's list is based on measuring in vitro the serum anticholinergic activity of drugs commonly used by older persons,${ }^{23}$ the Anticholinergic Drug Scale (ADS) is based on expert consensus ${ }^{24}$ and the 
Anticholinergic Risk Scale (ARS) on a literature review and expert opinion ${ }^{25}$. Although some studies have compared some anticholinergic criteria, ${ }^{4,5,26,27}$ there is still no international consensus regarding which of these lists would be applicable in research or in clinical practice.

The objectives of the current study were to investigate 1) how the use of DAPs according to any of the anticholinergic lists studied (Chew's list, ADS and ARS) is associated with participant characteristics, 2) how participant DAP use according to these anticholinergic lists is overlapping, and 3) how participant DAP use according to these anticholinergic lists and their combinations or the total number of DAPs according to any criteria (referred to as DAP burden) are associated with self-rated health, PWB, and mortality.

\section{Methods}

This study was part of a larger study ${ }^{28,29}$ that investigated the nutritional status, medication use, and associated factors of older persons in all long-term care facilities in Helsinki. During October and November 2011, cross-sectional data were collected from all older persons living permanently either in assisted living facilities or in nursing homes, including group homes for persons with dementia ( $\mathrm{n}=4449)$. Of all participants, 1097 were excluded because of patient refusal or dementia and without a close proxy to give informed consent. In addition, residents with severe dementia (score 3 on Clinical Dementia Rating (CDR) scale memory item) $)^{30}(\mathrm{n}=920)$ were excluded to include only those capable of responding to PWB. A total of 2432 participants thus remained in the study. Written informed consent was obtained from each participant or their closest proxy in case of significant cognitive decline (CDR 2). The study was approved by the Helsinki University Central Hospital Ethics Committee.

In each care unit, a trained nurse assessed the resident's status by retrieving background data from medical records on demographic factors and diagnoses (chronic conditions and acute illnesses) and performed the assessments and interviews according to a structured questionnaire. Each resident 
was assessed over the course of one day. All data concerning medication use was point prevalence on the same day.

Nutritional status was assessed by the Mini-Nutritional Assessment (MNA). ${ }^{31}$ Participants were respectively divided into "malnourished" (<17 points), "at risk for malnutrition" (17-23.5 points), and "well-nourished" ( $>23.5$ points). Cognitive function was evaluated by the memory item of the CDR scale (0-0.5, no or possible memory problems; 1 , mild memory loss; 2 , moderate memory loss), which is a validated method to assess dementia stage. ${ }^{30}$ The residents' ability to move was assessed by the question "Is the resident able to move inside?" (1=yes, $2=$ no, needs a stick or a walker, $3=$ no, needs another person's assistance, $4=$ no, unable to walk at all). Those in groups 1 and 2 were considered able to walk independently inside. Dependence in activities of daily living (ADL) was assessed by a 4-point scale according to the CDR "personal care" item (1=fully capable of self-care, $2=$ needs occasional prompting, $3=$ requires assistance in dressing, personal hygiene, and keeping of personal belongings, $4=$ requires much help with personal care; often incontinent). ${ }^{30}$ Those in groups 3 and 4 were considered as dependent on a caregiver's help. Participants' morbidity was assessed by the Charlson Comorbidity Index (CCI). ${ }^{32}$

Medication use was retrieved from medical charts during the assessment day. The resident was considered a regular drug user if the medical chart indicated a regular sequence for the drug dosage. Only regularly used DAPs were considered in determining the DAP burden. All medications were coded according to the Anatomical Therapeutic Chemical (ATC) Classification System of the World Health Organization (WHO) (WHO Collaborating Center for Drug Statistics Methodology). ${ }^{33}$ All DAPs used by the participants were listed and classified according to the following three anticholinergic lists: Chew's list, ${ }^{23} \mathrm{ADS},{ }^{24}$ and $\mathrm{ARS}^{25}$. According to Chew's list, 22 of 107 medications studied are demonstrated to have anticholinergic activity ranging from low $(+)$ to high (+++). The ADS rates 117 drugs as anticholinergic ranging from potentially anticholinergic (1) to markedly anticholinergic (3). The ARS classifies 49 drugs having 
anticholinergic potential ranging from moderate (1) to very strong (3). The three lists consist partly of different selection of anticholinergic drugs. The criteria for defining the anticholinergic potential or the defined burden also differ from each other. Thus, the anticholinergic activity and the anticholinergic burden is difficult to compare between the lists. In this study, the total number of DAPs is referred to as DAP burden.

QOL was evaluated by self-rated health and PWB questionnaire. Resident's subjective health (selfrated health) was evaluated by the following question: "How do you rate your current health status?" (1=healthy, $2=$ quite healthy, $3=$ unhealthy, $4=$ very unhealthy). Those responding "healthy" or "quite healthy" were considered as having good self-rated health. The PWB scale has been used in several studies since $1989 .{ }^{34-36}$ It has good test-retest reliability ${ }^{35}$, good prognostic validity ${ }^{34}$ and good concurrent validity with The World Health Organization Quality of Life - scale (WHOQOLBref $).{ }^{37}$ The residents were asked six questions as follows: 1 . Are you satisfied with your life? (yes/no); 2. Do you have zest for life? (yes/no); 3. Do you feel needed? (yes/no); 4. Do you have plans for the future? (yes/no); 5. Do you suffer from loneliness? (seldom or never/sometimes/often or always); 6. Do you feel depressed? (seldom or never/sometimes/often or always). The answers were then given 0 points ("no" in questions 1-4 and "often or always" in questions 5-6), 0.5 points ("sometimes" in questions 5-6) or 1 point ("yes" in questions 1-4 and "seldom or never" in questions 5-6). The PWB score was then generated by adding the points together and dividing the sum by the number of questions answered, thus yielding a score between 0 to 1 ( 0 represents the poorest and 1 the best well-being).

We divided the participants into DAP users and nonusers according to the use of at least one DAP included in one or more of the three lists mentioned above. We then generated a Venn diagram creating three overlapping groups of DAP lists as mentioned above (Figure 1). Only 14 drugs were considered as DAPs according to all three criteria. The number of DAPs used regularly by any criteria by each participant was counted. 
Mortality was retrieved from central registers over a 1-year follow -up.

148

149

150

151

152

153

154

155

156

157

158

\section{Statistical analyses}

Data are presented as absolute numbers, percentages for categorical variables and as means with standard deviations or ranges for continuous variables. The DAP users of according to any criteria were compared with nonusers with $\mathrm{X}^{2}$-test for categorical variables and t-test or permutation test for continuous variables.

The relationship between DAPs and mortality or PWB was analyzed using linear regression analysis and logistic models. Models included gender, age and Charlson index as covariates.

Shapiro-Wilk statistics were used to test the normality of variables. Stata 15.0, StataCorp LP (College Station, TX, USA) statistical package was used for the analysis.

\section{Results}

The Venn diagram (Figure 2) illustrates the DAP users' position in the various overlapping groups according to Chew`s list, ADS and ARS. Of all participants, 2079 (85\%) used at least one DAP according to any of the three criteria; $353(15 \%)$ did not use DAPs at all. Of DAP users, 1243 (60\%) used at least one DAP according to ARS, 1334 (64\%) used at least one DAP according to Chew's list, and 1652 (79\%) were administered at least one DAP according to ADS. Furthermore, of DAP users, 700 (34\%) used one or more DAPs according to only one list, 1379 (66\%) used DAPs according to at least two lists, and 771 (37\%) used one or more DAPs according to all three lists.

Compared with nonusers, DAP users were younger and had better cognition, but more often suffered from depression, other psychiatric disorders, and Parkinson disease. The CCI of DAP users was significantly higher than the respective value of nonusers $(2.4$ vs. $2.1, \mathrm{p}<0.001)$. Those using DAPs were more often at risk for malnutrition than nonusers $(\mathrm{p}=0.049)$. The mean number of 
regularly used drugs was significantly higher among DAP users than nonusers (9.3 vs. 5.4, p $<0.001)$. While DAP users suffered more often from dry mouth than nonusers $(\mathrm{p}=0.011)$, no difference between the groups was observed regarding constipation, diarrhea, or vomiting. No significant differences existed between the groups with respect to sex distribution, education, dependency on another person's help, or ability to walk inside (Table 1).

A larger proportion of participants not using DAPs considered their self-rated health as good compared to those using DAPs (70\% vs. 64\%, $\mathrm{p}=0.039)$. Those not using DAPs also had a significantly higher PWB score than those using DAPs (0.77 vs. $0.68, \mathrm{p}<0.001)$. There was no difference in mortality between users and nonusers (18\% vs. $20 \%, \mathrm{p}=0.44)$ (Table 1$)$.

In a further analysis regarding the relationship between DAP use and psychological well-being and mortality, the groups were compared to each other according to one, two, or three criteria of DAP use. Using DAPs according only to one criteria did not seem to affect PWB compared to nonusers, except for those using DAPs defined only by Chew's list, whose PWB was significantly lower than the PWB of nonusers. When using DAPs defined by two or three criteria, the participants did have significantly lower PWB compared to nonusers ( $\mathrm{p}<0.001$, adjusted for sex, age and comorbidity). There was no significant difference in mortality between the overlapping groups $(\mathrm{p}=0.41$, adjusted for sex, age, and comorbidity) (Figure 3). We further adjusted for number of medications, and the findings were essentially the same (data not shown).

When exploring the total burden of DAPs (i.e. the total number of DAPs according to any of the three criteria used), PWB decreased as a linear trend as the number of DAPs increased ( $\mathrm{p}<0.001$ for linearity, adjusted for sex, age, and comorbidity). The risk of mortality increased along with increasing number DAPs used ( $\mathrm{p}=0.006$ for linearity, adjusted for age, sex, and comorbidity) (Figure 4). We further adjusted for number of medications, and the findings were essentially the same (data not shown). 
194 We further performed sensitivity analyses by excluding all those with depression diagnosis. All the

195 findings were essentially the same (data not shown).

196

197 198

199

\section{Discussion}

Our study revealed that DAP use seems to have an inverse association with the PWB of older people living in long-term care facilities. Even though the DAP users were younger and had fewer cognitive problems than nonusers, their self-rated health and PWB was poorer than that of nonusers. DAP users suffered more often from depression and other psychiatric illnesses and had more comorbidities than nonusers. PWB decreased linearly as the number of DAPs increased. By investigating the overlapping of various criteria of DAPs among DAP users, we observed that neither the criteria alone nor a combination of two or three criteria predicted mortality. Instead, the total number of DAPs used according to any of these criteria (i.e. the total anticholinergic burden) was associated with increased risk of mortality.

Our study has several strengths and limitations. One strength of the study is the relatively large sample of older, frail people living in long-term care facilities in Helsinki. The data were collected by trained nurses by the same procedure in every participating unit. The information on demographic data, medication use and medical diagnoses were retrieved from each participant`s medical records. The mortality data were retrieved from central registers, which is $100 \%$ complete in Finland. The PWB scale is a well-validated tool even among older people with cognitive decline. Another strength was the use of three internationally well-known criteria in defining DAPs. Furthermore, we also focused on the burden of DAPs of each participant by counting the total number of DAPs according to any of the three lists.

One limitation of our study is its cross-sectional nature. Thus, it is not possible to draw definite conclusions between DAP use and PWB or mortality. The total time exposure of each individual medication is not known, as the data were collected as a point prevalence over one day and the 
follow-up time was one year. The original indication of the drugs is also unknown. Only regularly administered drugs were calculated in the total anticholinergic load, not drugs given pro re nata. All drugs were administered by nurses and adherence should thus be nearly $100 \%$. We also were unable to use the burden defined by the various scales as the three scales are not congruent in defining the anticholinergic burden; this can be considered a limitation. Our study demonstrates how relative the definitions and burden are according to various DAP scales. There may be confounding by indication bias regarding the use of DAPs and PWB. It is possible that PWB was poorer because of DAP use, or because the participants were given DAPs to manage some underlying disease (e.g. depression) that might have lowered their PWB score. However, our sensitivity analyses showed that the results were the same when those with depression were excluded. The results are consistent with our previous study from the same study population ${ }^{15}$ in which we observed that possible underlying depression did not explain the poorer PWB among DAP users.

DAP users were younger as in previous studies. ${ }^{38,39}$ DAP users suffered less often from cognitive problems compared to nonusers. In contrast to our previous study ${ }^{15}$, in the current study DAP users had more comorbidities according to the CCI than nonusers. One possible explanation is that the ADS contains a large number of very common drugs for coronary and heart diseases.

Our study demonstrates how the three scales differ from each other. Our combined list from three scales includes 138 DAPs but only 14 drugs are common in all scales. Of these 14 drugs, the most commonly used drugs were olanzapine and clozapine. The ADS scale has a high number of DAPs that are not included in other lists. Chew's list is quite compact but contains drugs frequently used by older people (e.g. mirtazapine and citalopram). Consistent with previous studies comparing various DAP scales, the scales seem to predict different outcomes from each other. ${ }^{12,40}$ However, to our knowledge there are no prior studies exploring how DAP use overlaps in real-life settings or how the number of DAPs used according to the three scales impacts on important outcomes. 
When comparing overlapping groups, we did not observe any significant differences in all-cause mortality between the groups, even after adjusting for age, sex, and CCI. This can be explained by the fact that the anticholinergic burden does not necessarily increase even though DAP use is defined by multiple criteria. Instead, when considering the total number of DAPs defined by any of 247 the three criteria, an association with increased mortality was demonstrated even after adjusting for 248 age, sex, and CCI. This is a new finding compared to most prior studies. ${ }^{6,13,17-19}$ Only one prior 249 scale, the Anticholinergic Cognitive Burden Scale $(A C B)^{41}$, has shown in several studies predictive 250 value on mortality. ${ }^{6,8,19,21}$ The ACB scale includes 88 DAPs, thus emphasizing that higher number of potential DAPs may have impact on mortality. There was a linear relationship between mortality and higher number of these drugs used.

253 To our knowledge, despite the importance of PWB of older persons living their last years in long254 term care facilities, there is still a lack of studies investigating the relationship between DAP use 255 and PWB. While Kolanowski et al. ${ }^{42}$ found no association between anticholinergic burden and 256 engagement in activity (an indicator of QOL in persons with dementia), Teramura-Grönblad et al. ${ }^{22}$ 257 and Aalto et al. ${ }^{15}$ found an association between PWB and DAP use. In an Australian study 258 assessing older people living in residential care facilities, an association was found between the use 259 of potentially harmful medicines defined by the Drug Burden Index and the self-reported QOL 260 measured with the Quality of Life-Alzheimer's disease questionnaire. ${ }^{43}$ A small American study 261 among community-dwelling older adults with dementia also revealed an association between DAP 262 use and lower health-related QOL. ${ }^{44}$ Our current study supports the earlier findings of the 263 association between DAPs and poorer PWB.

\section{Conclusions and Implications}

265 The use of DAPs seems to have a negative association with PWB of older and vulnerable people 266 living in institutionalized care. The DAP burden was linearly associated with poorer PWB. Neither 267 a single criterium alone nor a combination of two or three criteria predicted mortality. Instead, we 
268 found a weak, linear association between the total number of DAPs and increased mortality. The 269 most appropriate criteria for DAPs in assessing the anticholinergic load of older people remains 270 thus far unknown and further studies are needed.

272 The authors declare no conflicts of interest directly relevant to this report.

273 
Table 1. Participants' characteristics according to usage of drugs with anticholinergic properties (DAPs).

\begin{tabular}{|c|c|c|c|}
\hline & $\begin{array}{l}\text { Using no } \\
\text { DAPs } \\
n=353(15 \%)\end{array}$ & $\begin{array}{l}\text { Using one or } \\
\text { more DAPs } \\
n=2079(85 \%)\end{array}$ & $p$-value ${ }^{1}$ \\
\hline Female, n (\%) & $254(72)$ & $1568(75)$ & 0.16 \\
\hline Age, mean $\left(S D^{2}\right)$ & $86(7)$ & $84(8)$ & $<0.001$ \\
\hline Education $<8$ yrs, $\mathrm{n}(\%)$ & $201(57)$ & $1144(55)$ & 0.50 \\
\hline Depression, $\mathrm{n}(\%)$ & $37(10)$ & $489(24)$ & $<0.001$ \\
\hline Other psychiatric diagnosis, $\mathrm{n}(\%)$ & $18(5)$ & $291(14)$ & $<0.001$ \\
\hline Parkinson disease, $\mathrm{n}(\%)$ & $9(3)$ & $138(7)$ & 0.003 \\
\hline $\mathrm{CCl}^{3}$, mean $\left(\mathrm{SD}^{2}\right)$ & $2.1(1.4)$ & $2.4(1.5)$ & $<0.001$ \\
\hline $\mathrm{MNA}^{4}, \mathrm{n}(\%)$ & & & 0.049 \\
\hline $\begin{array}{l}\text { At risk for malnutrition (17-23.5 points), } n \\
\text { (\%) }\end{array}$ & $206(59)$ & $1353(65)$ & \\
\hline Moderate cognitive decline $(\mathrm{CDR} 2)^{5}, \mathrm{n}(\%)$ & $154(44)$ & $679(33)$ & $<0.001$ \\
\hline $\begin{array}{l}\text { Dependent for personal care (CDR personal } \\
\text { care class } 2-3)^{6}, \mathrm{n}(\%)\end{array}$ & $285(81)$ & $1724(83)$ & 0.32 \\
\hline Ability to walk inside, $\mathrm{n}(\%)$ & $117(50)$ & $1115(54)$ & 0.22 \\
\hline Number of drugs used regularly, mean $\left(\mathrm{SD}^{2}\right)$ & $5.4(2.9)$ & $9.3(3.4)$ & $<0.001$ \\
\hline Dry mouth, n (\%) & $36(10)$ & $319(15)$ & 0.011 \\
\hline Constipation, n (\%) & $125(35)$ & $719(35)$ & 0.76 \\
\hline Diarrhea, n (\%) & $32(9)$ & $253(12)$ & 0.094 \\
\hline Vomiting, n (\%) & $12(3)$ & $97(5)$ & 0.29 \\
\hline Self-rated health good, n (\%) & $246(70)$ & $1331(64)$ & 0.039 \\
\hline $\mathrm{PWB}^{7}$, mean $\left(\mathrm{SD}^{2}\right)$ & $0.77(0.23)$ & $0.68(0.26)$ & $<0.001$ \\
\hline One-year mortality, n (\%) & $71(20)$ & $374(18)$ & 0.44 \\
\hline
\end{tabular}

\footnotetext{
${ }^{1} \mathrm{p}$-value, adjusted for age, sex, comorbidity, CDR and dependency for personal care

${ }^{2} \mathrm{SD}=$ standard deviation

${ }^{3} \mathrm{CCl}=$ Charlson Comorbidity Index (Charlson et al. 1987) ${ }^{32}$

${ }^{4}$ MNA $=$ Mini-Nutritional Assessment (Vellas et al. 1999) ${ }^{31}$

${ }^{5} \mathrm{CDR}=$ Clinical Dementia Rating, "memory" item (Hughes et al. 1982) ${ }^{30}$

${ }^{6} \mathrm{CDR}$, "personal care" item (Hughes et al. 1982) ${ }^{30}$

${ }^{7}$ PWB $=$ psychological well-being (Routasalo et al. 2009) ${ }^{36}$
} 
Figure 1.

ADS (Carnahan et al. 2006)

117 anticholinergic drugs

prazolam, ampicillin, azathioprine, bromocriptine, brompheniramine, captopril, carbamazepine,

carbinoxamine, cefamandole, cefoxitin, cephalothin, chlordiazepoxide, chlorthalidone, clemastine,
clindamycin, clomipramine, clonazepam, clorazepate, codeine, cortisone, cycloserine, cyclosporine,

darifenacin, dexamethasone, diazepam, digitoxin, digoxin, dittiazem, dimenhydrinate, dipyridamole,

disopyramide dival roe sodium, estazolgm, famotidine, fentazenl flavoxate, flurazepam, fluticasone-

salmeterol, fluvoxamine, furosemide, gentamicin, hydralazine, hydrocortisone, isosorbide, isosorbide

dinitrate, isosoride mono itrate, ketotifen ophtalmic, lorazepam, brapine, meperidine,

methotrimeprazine, methylprednisolone, midazolam, molindone, morphine, nifedipine, nizatidine

orphenadrine, oxazeper

prednisolone, prednisone, procyclidine, propantheline, protriptyline, pyrilumine, scopolamine,

sertraline, theophylline, tramadol, triamcinolone, triamterene, triazolam, trihexyphenidy,

trimipramine, valproic acid, vancomycin, warfarin 


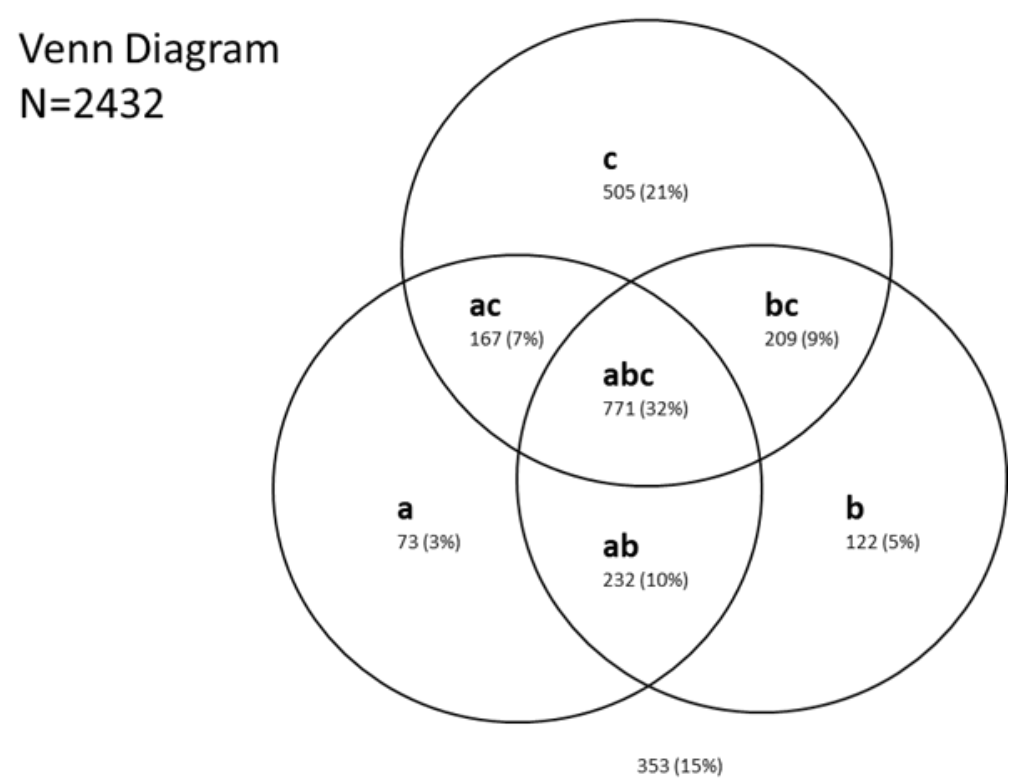

318 Figure 2.

319

320

321

322

323

324

325

326

327

328

329

330

331

332

333

334

335

336 
A

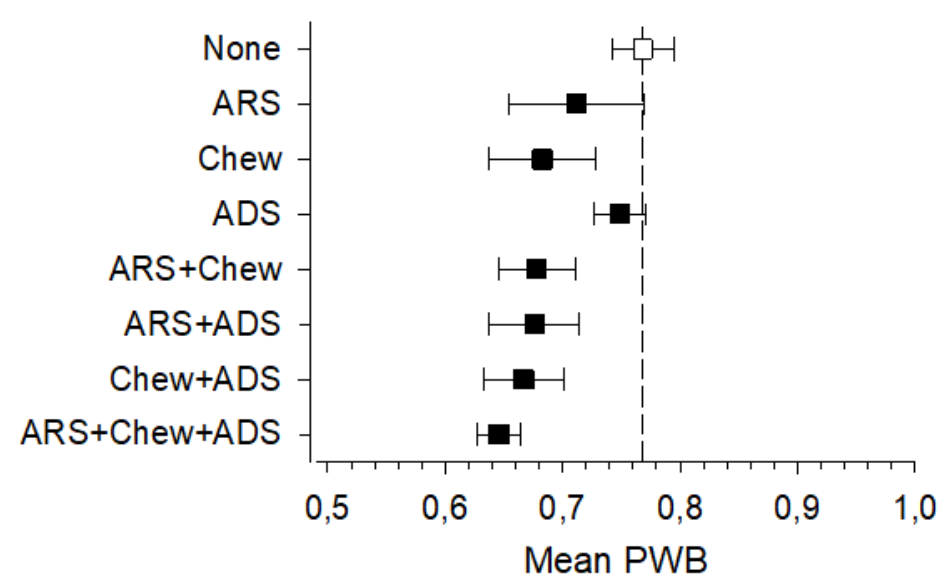

B

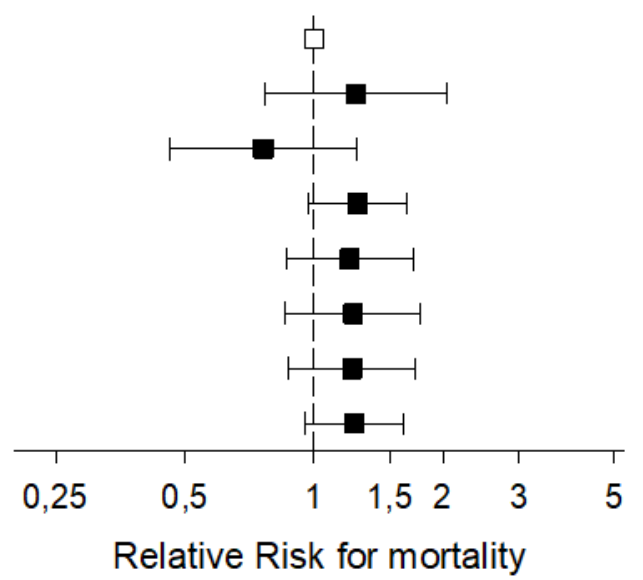

338

339

340

341

342

343

A

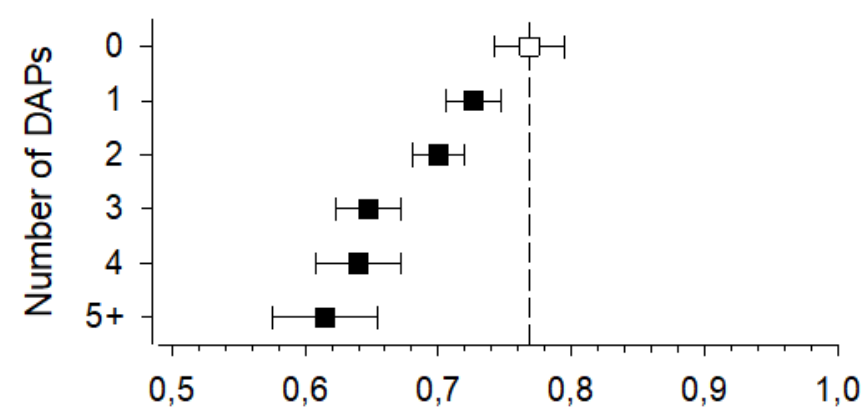

344

345

346

347

348

349

350

351

352

353

Figure 4.

Mean PWB
B

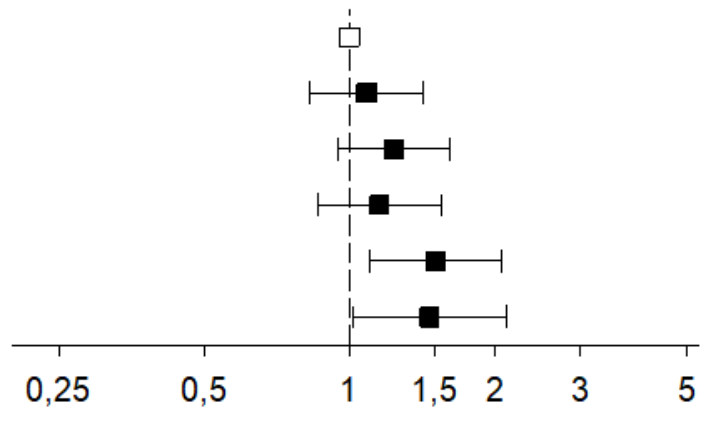

Relative Risk for mortality

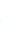


886

\section{7}

\section{Legends to figures}

Figure 1.

Venn diagram showing how the three scales on drugs with anticholinergic properties (DAP) overlap. Anticholinergic Risk Scale (ARS) ${ }^{25}$, Anticholinergic Drug Scale (ADS) ${ }^{24}$ and Chew's list ${ }^{23}$

Figure 2.

Venn diagram showing how the participants fell into groups using drugs with anticholinergic properties (DAP) in the overlapping groups of three DAP scales. DAP users N=2079 (85\%), nonusers $\mathrm{N}=353(15 \%)$

DAP users by: $\mathrm{a}=$ Anticholinergic Risk Scale (ARS) ${ }^{25} \mathrm{~b}=\mathrm{Chew}$ 's list (Chew) ${ }^{23} \mathrm{c}=$ Anticholinergic Drug Scale $(\mathrm{ADS}) ;{ }^{24} \mathrm{ab}=\mathrm{ARS}+\mathrm{Chew} ; \mathrm{ac}=\mathrm{ARS}+\mathrm{ADS} ; \mathrm{bc}=\mathrm{Chew}+\mathrm{ADS} ; \mathrm{abc}=\mathrm{ARS}+\mathrm{Chew}+\mathrm{ADS}$.

Figure 3.

A, Psychological well-being (PWB) in groups according to use of drugs with anticholinergic properties (DAP) by various criteria and how the overlapping groups predicted PWB $(\mathrm{p}<0.001)$. None=not using any DAP; Anticholinergic Risk Scale (ARS) ${ }^{25}$; Chew's list (Chew $)^{23}$; Anticholinergic Drug Scale (ADS) ${ }^{24}$

$B$, Relative risk for mortality is presented in respective groups $(p=0.41)$.

The relationships between DAPs and PWB or mortality were analyzed using linear regression analysis (mean) and logistic models (relative risk). Models included sex, age, and Charlson comorbidity index as covariates.

\section{Figure 4.}

A, Psychological well-being (PWB) in groups using no drugs with anticholinergic properties (DAPs) or using 1 to 5 or more DAPs. The higher the number of DAPs the lower is PWB ( $<<0.001$ for linearity).

$\mathrm{B}$, Relative risk for mortality is presented in respective groups. The number of DAPs has a linear relationship with mortality ( $\mathrm{p}=0.006$ for linearity).

The relationships between number of DAPs and PWB or mortality were analyzed using linear regression analysis (mean) and logistic models (relative risk). Models included sex, age, and Charlson comorbidity index as covariates. 


\section{References}

391

392

393

394

395

396

1. Tune LE. Anticholinergic effects of medication in elderly patients. J Clin Psychiatry. 2001;62 Suppl 21:11-14.

2. Mintzer J, Burns A. Anticholinergic side-effects of drugs in elderly people. J R Soc Med 2000;93:457-462

3. Chapter 13 Cholinergic transmission. In: Rang HP, Ritter JM, Flower RJ, Henderson G, editors. Rang and Dale’s Pharmacology. $8^{\text {th }}$ ed. Churchill Livingstone: Elsevier; 2016.

4. Villalba-Moreno AM, Alfaro-Lara ER, Pérez-Guerrero MC, et al. Systematic review on the use of anticholinergic scales in poly pathological patients. Arch Gerontol Geriatr 2016;62:1-8.

5. Salahudeen MS, Duffull SB, Nishtala PS. Anticholinergic burden quantified by anticholinergic risk scales and adverse outcomes in older people: A systematic review. BMC Geriatr 2015;15:31.

6. Ruxton K, Woodman RJ, Mangoni AA. Drugs with anticholinergic effects and cognitive impairment, falls and all-cause mortality in older adults: A systematic review and meta-analysis. $\mathrm{Br}$ J Clin Pharmacol 2015;80:209-220.

7. Campbell N, Boustani M, Limbil T, et al. The cognitive impact of anticholinergics: A clinical review. Clin Interv Aging 2009;4:225-233.

8. Fox C, Richardson K, Maidment ID, et al. Anticholinergic medication use and cognitive impairment in the older population: The medical research council cognitive function and ageing study. J Am Geriatr Soc 2011;59:1477-1483.

9. Gerretsen P, Pollock BG. Cognitive risks of anticholinergics in the elderly. Aging Health. 2013;9:159-166.

10. Uusvaara J, Pitkala KH, Kautiainen H, et al. Detailed cognitive function and use of drugs with anticholinergic properties in older people: A community-based cross-sectional study. Drugs Aging 2013;30:177-182. 
11. Salahudeen MS, Hilmer SN, Nishtala PS. Comparison of anticholinergic risk scales and

415

416

417 associations with adverse health outcomes in older people. J Am Geriatr Soc 2015;63:85-90. 12. Hsu WH, Wen YW, Chen LK, Hsiao FY. Comparative associations between measures of anticholinergic burden and adverse clinical outcomes. Ann Fam Med 2017;15(6):561-569.

13. Kumpula EK, Bell JS, Soini H, Pitkälä KH. Anticholinergic drug use and mortality among residents of long-term care facilities: A prospective cohort study. J Clin Pharmacol 2011;51:256263.

14. Palmer JB, Albrecht JS, Park Y, et al. Use of drugs with anticholinergic properties among nursing home residents with dementia: A national analysis of medicare beneficiaries from 2007 to 2008. Drugs Aging 2015;32:79-86.

15. Aalto UL, Roitto HM, Finne-Soveri H, et al. Use of anticholinergic drugs and its relationship with psychological well-being and mortality in long-term care facilities in Helsinki. J Am Med Dir Assoc 2018;19:511-515.

16. Dauphinot V, Mouchoux C, Veillard S, et al. Anticholinergic drugs and functional, cognitive impairment and behavioral disturbances in patients from a memory clinic with subjective cognitive decline or neurocognitive disorders. Alzheimers Res Ther 2017;9:58.

17. Luukkanen MJ, Uusvaara J, Laurila JV, et al. Anticholinergic drugs and their effects on delirium and mortality in the elderly. Dement Geriatr Cogn Dis Extra 2011;1:43-50.

18. Wilson NM, Hilmer SN, March LM, et al. Associations between drug burden index and mortality in older people in residential aged care facilities. Drugs Aging 2012;29:157-165.

19. Fox C, Smith T, Maidment I, et al. Effect of medications with anti-cholinergic properties on cognitive function, delirium, physical function and mortality: A systematic review. Age Ageing 2014;43:604-615. 
20. Gutiérrez-Valencia M, Martínez-Velilla N, Vetrano DL, et al. Anticholinergic burden and health outcomes among older adults discharged from hospital: results from the CRIME study. Eur J Clin Pharmacol 2017;73:1467-1474.

21. Myint PK, Fox C, Kwok CS, et al. Total anticholinergic burden and risk of mortality and cardiovascular disease over 10 years in 21,636 middle-aged and older men and women of EPICNorfolk prospective population study. Age Ageing 2015;44:219-225.

22. Teramura-Grönblad M, Muurinen S, Soini H, et al. Use of anticholinergic drugs and cholinesterase inhibitors and their association with psychological well-being among frail older adults in residential care facilities. Ann Pharmacother 2011;45:596-602.

23. Chew ML, Mulsant BH, Pollock BG, et al. Anticholinergic activity of 107 medications commonly used by older adults. J Am Geriatr Soc 2008;56:1333-1341.

24. Carnahan RM, Lund BC, Perry PJ, et al. The anticholinergic drug scale as a measure of drugrelated anticholinergic burden: Associations with serum anticholinergic activity. J Clin Pharmacol 2006;46:1481-1486.

25. Rudolph JL, Salow MJ, Angelini MC, McGlinchey RE. The anticholinergic risk scale and anticholinergic adverse effects in older persons. Arch Intern Med 2008;168:508-513.

26. Duran CE, Azermai M, Vander Stichele RH. Systematic review of anticholinergic risk scales in older adults. Eur J Clin Pharmacol 2013;69:1485-1496.

27. Naples JG, Marcum ZA, Perera S, et al. Concordance between anticholinergic burden scales. J Am Geriatr Soc 2015;63:2120-2124.

28. Suominen M, Muurinen S, Routasalo P, et al. Malnutrition and associated factors among aged residents in all nursing homes in helsinki. Eur J Clin Nutr 2005;59:578-583.

29. Suominen MH, Sandelin E, Soini H, Pitkala KH. How well do nurses recognize malnutrition in elderly patients? Eur J Clin Nutr 2009;63:292-296. 
30. Hughes CP, Berg L, Danziger WL, et al. A new clinical scale for the staging of dementia. Br J Psychiatry. 1982;140:566-572.

31. Vellas B, Guigoz Y, Garry PJ, et al. The mini nutritional assessment (MNA) and its use in grading the nutritional state of elderly patients. Nutrition. 1999;15:116-122.

32. Charlson ME, Pompei P, Ales KL, MacKenzie CR. A new method of classifying prognostic comorbidity in longitudinal studies: Development and validation. J Chronic Dis. 1987;40:373-383.

33. WHO Collaborating Centre for Drug Statistics Methodology. The Anatomical Therapeutical Chemical Classification System. Norwegian Institute of Public Health. Available at: https://www.whocc.no/atc_ddd_index/. Accessed September 20, 2010.

34. Pitkala KH, Laakkonen ML, Strandberg TE, Tilvis RS. Positive life orientation as a predictor of 10-year outcome in an aged population. J Clin Epidemiol 2004;57:409-414.

35. Savikko N, Routasalo P, Tilvis RS, et al. Loss of parents in childhood - associations with depression, loneliness, and attitudes towards life in older finnish people. Int J Older People Nurs 2006;1:17-24.

36. Routasalo PE, Tilvis RS, Kautiainen H, Pitkala KH. Effects of psychosocial group rehabilitation on social functioning, loneliness and well-being of lonely, older people: randomized controlled trial. J Adv Nurs 2009;65:297-305.

37. Huusko T, Kautiainen H, Pitkala H. WHOQOL-Bref and 150 in measuring quality-of-life among older caregivers. In: Huusko T, Strandberg T, Pitkala K, editors. Can Older People's Quality of Life Be Measured? [Finnish]. Helsinki, Finland: The Central Union for the Welfare of the Aged; 2006. p. 76-90.

38. Chatterjee S, Mehta S, Sherer JT, Aparasu RR. Prevalence and predictors of anticholinergic medication use in elderly nursing home residents with dementia: Analysis of data from the 2004 national nursing home survey. Drugs Aging 2010;27:987-997. 
39. Landi F, Dell'Aquila G, Collamati A, et al. Anticholinergic drug use and negative outcomes

486 among the frail elderly population living in a nursing home. J Am Med Dir Assoc 2014;15:825-829.

487 40. Lertxundi U, Domingo-Echaburu S, Hernandez R, et al. Expert-based drug lists to measure

488 anticholinergic burden: similar names, different results. Psychogeriatrics 2013;13:17-24.

489 41. Boustani M, Campbell N, Munger S, et al. Impact of anticholinergics on the aging brain: a

490 review and practical application. Aging Health 2008;4:311-320.

491 42. Kolanowski A, Fick DM, Campbell J, et al. A preliminary study of anticholinergic burden and 492 relationship to a quality of life indicator, engagement in activities, in nursing home residents with 493 dementia. J Am Med Dir Assoc 2009;10:252-257.

494 43. Bosboom PR, Alfonso H, Almeida OP, Beer C. Use of potentially harmful medications and 495 health-related quality of life among people with dementia living in residential aged care 496 facilities. Dement Geriatr Cogn Dis Extra 2012;2:361-371.

497 44. Sura SD, Carnahan RM, Chen H, Aparasu RR. Anticholinergic drugs and health-related quality 498 of life in older adults with dementia. J Am Pharm Assoc (2003) 2015;55:282-287. 